\title{
A Simple Proof for a Theorem of Nagel and Schenzel
}

\author{
Duong Thi Huong* \\ Department of Mathematics, Thang Long University, Hanoi, Vietnam \\ Received 12 December 2017 \\ Revised 25 December 2017; Accepted 28 December 2017
}

\begin{abstract}
Nagel-Schenzel's isomorphism that has many applications was proved by using spectral sequence theory. In this short note, we present a simple proof for the theorem of Nagel and Schenzel.

Keywords: Local cohomology, filter regular sequence.
\end{abstract}

\section{Introduction}

Throughout this paper, let $R$ be a commutative Noetherian ring, $M$ a finitely genrated $R$-module and $I$ an ideal of $R$. Local cohomology $H_{I}^{i}(M)$ introduced by Grothendieck, is an important tool in both algebraic geometry and commutative algebra (cf. [2]). Moreover, the notion of $I$-filter regular sequences on $M$ is an useful technique in study local cohomology. In [4] Nagel and Schenzel proved the following theorem (see also [1]).

Theorem 1.1. Let I be an ideal of a Noetherian ring $R$ and $M$ a finitely generated $R$-module. Let $x_{1}, \ldots, x_{n}$ an I-filter regular sequence of $M$. Then we have

$$
H_{I}^{i}(M) \cong \begin{cases}H_{\left(x_{1}, \ldots, x_{t}\right)}^{i}(M) & \text { if } i<t \\ H_{I}^{i-t}\left(H_{\left(x_{1}, \ldots, x_{t}\right)}^{i}(M)\right) & \text { if } i \geq t .\end{cases}
$$

The most important case of Theorem 1.1 is $i=t$, and $H_{I}^{t}(M) \cong H_{I}^{0}\left(H_{\left(x_{1}, \ldots, x_{t}\right)}^{t}(M)\right)$ is a submodule of $H_{\left(x_{1}, \ldots, x_{t}\right)}^{t}(M)$. Recently, many applications of this fact have been found [3,5]. It should be noted that Nagel-Schenzel's theorem was proved by using spectral sequence theory. The aim of this short note is to give a simple proof for Theorem 1.1 based on standard argument on local cohomology [2].

\section{Proofs}

Firstly, we recall the notion of $I$-filter regular sequence on $M$.

\footnotetext{
*Corresponding author. Tel.: 84-983602625.

Email: duonghuongtlu@gmail.com

https//doi.org/ 10.25073/2588-1124/vnumap.4249
} 
Definition 2.1. Let $M$ be a finitely generated module over a local ring $(R, \mathrm{~m}, \mathrm{k})$ and let $x_{1}, \ldots, x_{t} \in I$ be a sequence of elements of $R$. Then we say that $x_{1}, \ldots, x_{t}$ is a I-filter regular sequence on $M$ if the following conditions hold:

$$
\operatorname{Supp}\left(\left(\left(x_{1}, \ldots, x_{i-1}\right) M: x_{i}\right) /\left(x_{1}, \ldots, x_{i-1}\right) M \subseteq V(I)\right.
$$

for all $i=1, \ldots, t$, where $V(I)$ denotes the set of prime ideals containing $I$. This condition is equivalent to $x_{i} \notin \mathfrak{p}$ for all $\mathfrak{p} \in A s s_{R} M /\left(x_{1}, \ldots, x_{i-1}\right) M \backslash V(I)$ and for all $i=1, \ldots, t$.

Remark 2.2. It should be noted that for any $t \geq 1$ we always can choose a $I$-filter regular sequence $x_{1}, \ldots, x_{t}$ on $\mathrm{M}$. Indeed, by the prime avoidance lemma we can choose $x_{1} \in I$ and $x_{1} \notin \mathfrak{p}$ for all $\mathfrak{p} \in A s s_{R} R \backslash V(I)$. For $i>1$ assume that we have $x_{1}, \ldots, x_{i-1}$, then we choose $x_{i} \in I$ and $x_{i} \notin \mathfrak{p}$ for all $\mathfrak{p} \in A s s_{R} R /\left(x_{1}, \ldots, x_{i-1}\right) \backslash V(I)$ by the prime avoidance lemma again. For more details, see [1, Section 2].

The $I$-filter regular sequence can be seen as a generalization of the well-known notion of regular sequence (cf. [4, Proposition 2.2]).

Lemma 2.3. A sequence $x_{1}, \ldots, x_{t} \in I$ is an $I$-filter regular sequence on $M$ if and only if for all $\mathfrak{p} \in \operatorname{Spec}(R) \backslash V(I)$, and for all $i \leq t$ such that $x_{1}, \ldots, x_{i} \in \mathfrak{p}$ we have $\frac{x_{1}}{1}, \ldots, \frac{x_{t}}{1}$ is an $M_{\mathfrak{p}}$-sequence.

Corollary 2.4. Let $x_{1}, \ldots, x_{t} \in I$ be an $I$-filter regular sequence on $M$. Then $H_{\left(x_{1}, \ldots, x_{t}\right)}^{i}(M)$ is $I$-torsion for all $i<t$.

Proof. For each $\mathfrak{p} \in \operatorname{Spec}(R) \backslash V(I)$ we have either $\left(x_{1}, \ldots x_{t}\right) R_{\mathfrak{p}}=R_{\mathfrak{p}}$ or $x_{1}, \ldots, x_{t}$ is an $M_{\mathfrak{p}}$-regular sequence by Lemma 2.3. For the first case we have

$$
\left(H_{\left(x_{1}, \ldots, x_{t}\right)}^{i}(M)\right)_{\mathfrak{p}} \cong H_{\left(x_{1}, \ldots, x_{t}\right) R_{\mathfrak{p}}}^{i}\left(M_{\mathfrak{p}}\right)=0
$$

for all $i \geq 0$. For the second case we have

$$
\left(H_{\left(x_{1}, \ldots, x_{t}\right)}^{i}(M)\right)_{\mathfrak{p}} \cong H_{\left(x_{1}, \ldots, x_{t}\right) R_{\mathfrak{p}}}^{i}\left(M_{\mathfrak{p}}\right)=0
$$

for all $i<t$ by the Grothendieck vanishing theorem [2, Theorem 6.2.7]. Therefore we have $\left(H_{\left(x_{1}, \ldots, x_{t}\right)}^{i}(M)\right)_{\mathfrak{p}} \cong 0$ for all $i<t$ and for all $\mathfrak{p} \in \operatorname{Spec}(R) \backslash V(I)$. So $H_{\left(x_{1}, \ldots, x_{t}\right)}^{i}(M)$ is $I-$ torsion for all $i<t$.

It is well-known that local cohomology $H_{\left(x_{1}, \ldots, x_{t}\right)}^{i}(M)$ agrees with the $i$-th cohomology of the Čech complex with respect to the sequence $x_{1}, \ldots, x_{t}$

$$
0 \rightarrow M \stackrel{d^{0}}{\rightarrow} \bigoplus_{i} M_{x_{i}} \stackrel{d^{1}}{\rightarrow} \bigoplus_{i<j} M_{x_{i} x_{j}} \stackrel{d^{2}}{\rightarrow} \cdots \stackrel{d^{t-1}}{\longrightarrow} M_{x_{1} \ldots x_{t}} \rightarrow 0
$$

The following simple fact is the crucial key for our proof.

Lemma 2.5. Let $x \in I$ be any element of $R$. Then $H_{I}^{i}\left(M_{x}\right)=0$ for all $i \geq 0$.

Proof. Obiviously the multiplication map $M_{x} \stackrel{x}{\rightarrow} M_{x}$ is an isomorphism. It induces isomorphism maps $H_{I}^{i}\left(M_{x}\right) \stackrel{x}{\rightarrow} H_{I}^{i}\left(M_{x}\right)$ for all $i \geq 0$. But $H_{I}^{i}\left(M_{x}\right)$ is $I$ - torsion, so it $(x)$-torsion since $x \in I$. Therefore $H_{I}^{i}\left(M_{x}\right)=0$ for all $i \geq 0$.

We are ready to prove the theorem of Nagel and Schenzel. 
Proof of Theorem 1.1. We set $(\underline{x})$ the ideal generated by $x_{1}, \ldots, x_{t}$. Let $C_{j}$ the $j$-th chain of $\breve{C}$ ech

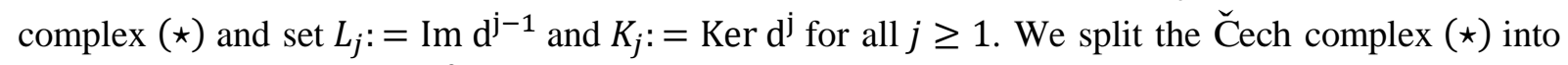
short exact sequences $0 \rightarrow H_{(\underline{x})}^{0}(M) \rightarrow M \rightarrow L_{1} \rightarrow 0 \quad\left(A_{0}\right)$

$$
\begin{aligned}
& 0 \rightarrow L_{1} \rightarrow K_{1} \rightarrow H_{(\underline{x})}^{1}(M) \rightarrow 0 \\
& 0 \rightarrow K_{1} \rightarrow C_{1} \rightarrow L_{2} \rightarrow 0 \\
& \ldots \\
& 0 \rightarrow L_{j} \rightarrow K_{j} \rightarrow H_{(\underline{x})}^{j}(M) \rightarrow 0 \\
& 0 \rightarrow K_{j} \rightarrow C_{j} \rightarrow L_{j+1} \rightarrow 0 \\
& \ldots \\
& 0 \rightarrow L_{t-1} \rightarrow K_{t-1} \rightarrow H_{(\underline{x})}^{t-1}(M) \rightarrow 0 \quad\left(B_{1}\right) \\
& 0 \rightarrow K_{t-1} \rightarrow C_{t-1} \rightarrow L_{t} \rightarrow 0 \\
& 0 \rightarrow L_{t} \rightarrow M_{x_{1} \ldots x_{t}} \rightarrow H_{(\underline{x})}^{t}(M) \rightarrow 0 . \quad\left(A_{t-1}\right)
\end{aligned}
$$

By Lemma 2.3 we have $H_{I}^{i}\left(C_{j}\right)=0$ for all $i \geq 0$ and all $j \geq 1$ Since $L_{j}$ and $K_{j}$ are submodules of $C_{j}$ for all $j \geq 1$ we have $H_{I}^{0}\left(L_{j}\right) \cong H_{I}^{0}\left(K_{j}\right)=0$ for all $j \geq 1$. We also note that $H_{(\underline{x})}^{j}(M)$ is $I$ torsion for all $j<t$ by Corollary 2.4 , so $H_{I}^{0}\left(H_{(\underline{x})}^{j}(M)\right)=H_{(\underline{x})}^{j}(M)$ and $H_{I}^{i}\left(H_{(\underline{x})}^{j}(M)\right) \cong 0$ for all $j<t$ and for all $i \geq 1$.

Now applying the functor $H_{I}^{i}(-)$ to the short exact sequence $\left(A_{0}\right)$ and using the above observations we have

$$
H_{I}^{0}(M) \cong H_{(\underline{x})}^{0}(M)
$$

and

$$
H_{I}^{i}(M) \cong H_{I}^{i}\left(L_{1}\right)
$$

for all $i \geq 1$.

For each $j=1, \ldots, t-1$, applying the local cohomology functor $H_{I}^{i}(-)$ to the short exact sequence $\left(A_{j}\right)$ we have $H_{I}^{1}\left(K_{j}\right)=0$ and the isomorphism

$$
H_{I}^{i}\left(L_{j+1}\right) \cong H_{I}^{i+1}\left(K_{j}\right) \quad\left(C_{j}\right)
$$

for all $i \geq 1$. Furthermore, if we apply $H_{I}^{i}(-)$ for the short exact sequence $\left(B_{j}\right)$, then we get the short exact sequence

$$
0 \rightarrow H_{(\underline{x})}^{j}(M) \rightarrow H_{I}^{1}\left(L_{j}\right) \rightarrow H_{I}^{1}\left(K_{j}\right) \rightarrow 0,
$$

and the isomorphism

$$
H_{I}^{i}\left(L_{j}\right) \cong H_{I}^{i}\left(K_{j}\right) \quad\left(D_{j}\right)
$$

for all $i \geq 2$. Note that $H_{I}^{1}\left(K_{j}\right)=0$ as above, so

$$
H_{(\underline{x})}^{j}(M) \cong H_{I}^{1}\left(L_{j}\right)
$$


We next show that $H_{I}^{i}(M) \cong H_{(\underline{x})}^{i}(M)$ for all $i=1, \ldots, t-1$. Indeed, using isomorphisms (1), (2), $\left(C_{j}\right)$ and $\left(D_{j}\right)$ consecutively, we have

$$
H_{I}^{i}(M) \stackrel{(1)}{\cong} H_{I}^{i}\left(L_{1}\right) \stackrel{\left(\mathrm{D}_{1}\right)}{\cong} H_{I}^{i}\left(K_{1}\right) \stackrel{\left(\mathrm{C}_{1}\right)}{\cong} H_{I}^{i-1}\left(L_{2}\right) \stackrel{\left(\mathrm{D}_{2}\right)}{\cong} \cdots \stackrel{\left(\mathrm{C}_{\mathrm{i}-1}\right)}{\cong} H_{I}^{1}\left(L_{i}\right) \stackrel{(2)}{\cong} H_{(\underline{x})}^{i}(M) .
$$

Therefore, we have showed the first case of Nagel-Schenzel's isomorphism $H_{I}^{i}(M) \cong H_{(\underline{x})}^{i}(M)$ for all $i=0, \ldots, t-1$. Finally, for $i \geq t$ by similar arguments we have

$$
H_{I}^{i}(M) \stackrel{(1)}{\cong} H_{I}^{i}\left(L_{1}\right) \stackrel{\left(\mathrm{D}_{1}\right)}{\cong} H_{I}^{i}\left(K_{1}\right) \stackrel{\left(\mathrm{C}_{1}\right)}{\cong} H_{I}^{i-1}\left(L_{2}\right) \stackrel{\left(\mathrm{D}_{2}\right)}{\cong} \cdots \stackrel{\left(\mathrm{C}_{\mathrm{t}-1}\right)}{\cong} H_{I}^{i-t+1}\left(L_{t}\right) .
$$

On the other hand, by applying the functor $H_{I}^{i}(-)$ to the short exact sequence $\left(B_{t}\right)$ we have $H_{I}^{i-t}\left(H_{(\underline{x})}^{t}(M)\right) \cong H_{I}^{i-t+1}\left(L_{t}\right)$

for all $i \geq t$. Thus $H_{I}^{i}(M) \cong H_{I}^{i-t}\left(H_{(\underline{x})}^{t}(M)\right)$ for all $i \geq t$, and we finish the proof.

\section{References}

[1] J. Asadollahi and P. Schenzel, Some results on associated primes of local cohomology modules, Japanese J. Mathematics 29 (2003), 285-296.

[2] M. Brodmann and R.Y. Sharp, Local cohomology: an algebraic introduction with geometric applications, Cambridge University Press, 1998.

[3] H. Dao and P.H. Quy, On the associated primes of local cohomology, Nagoya Math. J., to appear.

[4] U. Nagel and P. Schenzel, Cohomological annihilators and Castelnuovo-Mumford regularity, in Commutative algebra: Syzygies, multiplicities, and birational algebra, Contemp. Math. 159 (1994), Amer. Math. Soc. Providence, R.I., 307-328.

[5] P.H. Quy and K. Shimomoto, F -injectivity and Frobenius closure of ideals in Noetherian rings of characteristic $\mathrm{p}$ $>$ 0, Adv. Math. 313 (2017), 127-166. 